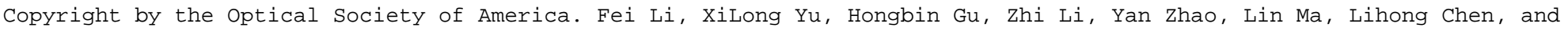

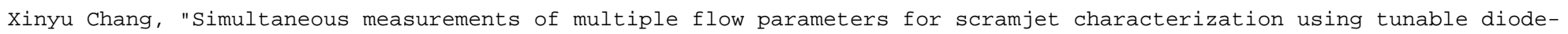
laser sensors," Appl. Opt. 50, 6697-6707 (2011); doi: 10.1364/A0.50.006697

\title{
Simultaneous measurements of multiple flow parameters for scramjet characterization using tunable diode-laser sensors
}

\author{
Fei Li, ${ }^{1}$ XiLong Yu, ${ }^{1, \star}$ Hongbin Gu, ${ }^{1}$ Zhi Li, ${ }^{1}$ Yan Zhao, ${ }^{2}$ Lin Ma, ${ }^{3}$ \\ Lihong Chen, ${ }^{1}$ and Xinyu Chang ${ }^{1}$ \\ ${ }^{1}$ State Key Laboratory of High Temperature Gas Dynamics, Institute of Mechanics, \\ Chinese Academy of Sciences, Beijing 100190, China \\ ${ }^{2}$ Air Products \& Chemicals Inc., Allentown, Pennsylvania 18195, USA \\ ${ }^{3}$ Department of Aerospace and Ocean Engineering, Virginia Tech, Blacksburg, Virginia 24061, USA \\ *Corresponding author: xlyu@ @imech.ac.cn
}

Received 1 July 2011; revised 25 September 2011; accepted 3 October 2011; posted 11 October 2011 (Doc. ID 150316); published 20 December 2011

\begin{abstract}
This paper reports the simultaneous measurements of multiple flow parameters in a scramjet facility operating at a nominal Mach number of 2.5 using a sensing system based on tunable diode-laser absorption spectroscopy (TDLAS). The TDLAS system measures velocity, temperature, and water vapor partial pressure at three different locations of the scramjet: the inlet, the combustion region near the flame stabilization cavity, and the exit of the combustor. These measurements enable the determination of the variation of the Mach number and the combustion mode in the scramjet engine, which are critical for evaluating the combustion efficiency and optimizing engine performance. The results obtained in this work clearly demonstrated the applicability of TDLAS sensors in harsh and high-speed environments. The TDLAS system, due to its unique virtues, is expected to play an important role in the development of scramjet engines. (C) 2011 Optical Society of America
\end{abstract}

OCIS codes: $\quad 120.1740,280.3420,300.1030,300.6260$.

\section{Introduction}

Over the past 50 years, the scramjet has attracted considerable research interest due to its potential as an air-breathing propulsion device for future hypersonic vehicles $[\underline{1}, 2]$. Past studies have identified several key challenges in optimizing scramjet combustors, which include achieving well-controlled dual-mode operation and high combustion efficiency [3-7]. The ability to measure the Mach number in the combustor is highly desirable to address these challenges. Therefore, researchers have developed various diagnostics for such measurements. Diagnostics based on tunable diode-laser absorption spectroscopy (TDLAS) have emerged as an attractive

0003-6935/11/366697-11\$15.00/0

(C) 2011 Optical Society of America technique, due to its robustness, relatively simple implementation, and capability to simultaneously measure multiple flow parameters in high-speed flows (including velocity, temperature, pressure, species concentration, and mass flow rate) [8-10].

Consequently, many variations of the TD $\bar{L} \overline{A S}$ diagnostic have been successfully demonstrated for in situ measurements of various flow parameters in scramjet test facilities [11-17]. For instance, Upschultep et al. [11] measured velocity, static temperature, and water vapor concentration at the inlet of the combustor in a direct-connected test facility. Griffiths and Houwing [13] measured temperature and water vapor concentration in the expanding exit region of the combustor in a shock tunnel at a $20 \mathrm{kHz}$ repetition rate. Liu et al. [14] measured the temperature and water partial pressure at eight fixed vertical positions at the exit section using both direct 
absorption and a wavelength modulation strategy. Preliminary estimation was made for the nonuniform temperature distribution along the laser path using three water absorption lines. Gruber et al. [15] used two water absorption lines to measure water vapor concentration and static temperature at nine fixed vertical positions near the combustor exit. Lindstrom et al. [16] studied the shock train structure in a rectangular isolator using multiple line-of-sight absorption measurements. Rieker et al. [17] demonstrated a three-wavelength tunable diode-laser temperature sensor to study combustion instabilities.

These past efforts have demonstrated the applicability of a TDLAS diagnostic in scramjet engines. However, these past measurements focused mostly on one location in the engine, the exit, while measurements at multiple locations (the inlet, the combustion region, and the exit, as shown in Fig. 1) are needed to quantify the performance of the engine. Specifically, these measurements include the (1) velocity measurements in the combustion region and the exit, and (2) temperature measurements in the combustion region. These measurements combined will yield the local Mach number, which is critical in distinguishing the combustion mode. Furthermore, it is highly desirable to make these measurements at each location with spatial resolution along the height (i.e., the $y$ direction shown in Fig. 1), because prior TDLAS measurements have shown nonuniform profiles of both temperature and water concentration [15] in the combustion region. Such spatially resolved measurements can be used to infer the enthalpy flux at each location, and, consequently, the combustion efficiency can be calculated with the enthalpy fluxes at the inlet and the exit of the combustor.

Based on the above considerations, a multichannel TDLAS system was developed in this work to address the diagnostic needs mentioned above. The TDLAS system uses the absorption spectroscopy of water vapor and the Doppler shift to measure temperature, water vapor concentration, and velocity. These parameters were simultaneously measured at three different locations (at the inlet, in the com-

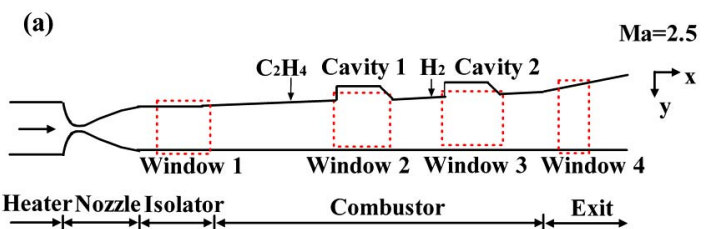

(b)

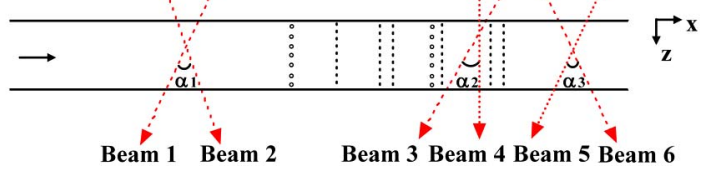

Fig. 1. (Color online) Schematic of (a) the direct-connected scramjet test facility (side view, not scaled in the $x$ direction), and (b) the laser beams' collocation (top view). bustor, and at the exit) by splitting the TDLAS system into multiple channels using fiber optics. The distributions of these parameters along the height at each measurement location were obtained by scanning the TDLAS system. The measurement technique is described in more detail in Section 2. Section 3 describes the implementation of the TDLAS system and the scramjet test facility, followed by results and analysis in Section 4 . Section 5 discusses the calculation of combustion efficiency and measurement uncertainty, and, finally, Section $\underline{6}$ summarizes the paper.

\section{Measurement Technique}

This section provides a brief review of the theory of TDLAS and its application for measuring velocity, temperature, and species concentration. More detailed discussion can be found in $[10,18,19]$. According to the Beer-Lambert law of absorption, the absorbance at a specific transition/wavelength depends on temperature, the concentration of the absorbing species, and the pressure. Measurement of absorbance at two carefully selected wavelengths can be used to simultaneously infer the temperature and concentration of the absorbing species if pressure is known. More specifically, temperature is inferred from the ratio of the measured integrated absorbance of the transitions, and partial pressure of the absorbing species $\left(\mathrm{H}_{2} \mathrm{O}\right.$ vapor in this work) is inferred from the integrated absorbance at either transition after temperature is determined. Flow velocity is inferred by measuring the Doppler frequency shift of the absorption peak $[\underline{8}, \underline{10}, \underline{20}]$ according to the following equation:

$$
\Delta \nu_{\text {Doppler }}=\frac{V}{c} \nu_{0} \cos \theta
$$

where $\Delta \nu_{\text {Doppler }}$ represents the Doppler frequency shift of the absorption peak, $V$ is the velocity of flow, $c$ is the speed of light, $\nu_{0}$ is the line-center frequency of the transition, and $\theta$ is the angle between laser propagation and flow direction.

A key aspect of implementing this so-called twowavelength TDLAS involves the selection of the optimal transitions, and the transitions used in this work are shown in Table 1 . They were selected based on the spectroscopic parameters provided in the HITRAN 2008 database [21], with the parameters of some transitions replaced by the measured values reported in $[14,22]$. These transitions were selected considering the criteria discussed in [23]. First, they

Table 1. Spectroscopic Line Parameters Used in the Current Study

\begin{tabular}{lccc}
\hline & & & Frequency \\
Transition & $\nu_{0}\left(\mathrm{~cm}^{-1}\right)$ & $\begin{array}{c}\text { Line Strength } \\
S\left(\mathrm{~atm}^{-1} \mathrm{~cm}^{-2}\right)\end{array}$ & $\begin{array}{c}\text { Energy } \\
E^{\prime \prime}\left(\mathrm{cm}^{-1}\right)\end{array}$ \\
\hline 1 & 7185.597 & $1.96 \times 10^{-2}$ & 1045.058 \\
2 & $7444.35+7444.37$ & $1.10 \times 10^{-3}$ & 1774.751 \\
& $($ combined) & & 1806.670 \\
\hline
\end{tabular}


provide sufficient absorption strength to ensure high signal-to-noise ratio (SNR) [24]. Second, they provide suitable low-state energy to ensure high sensitivity in the temperature range of interest in this work.

The TDLAS measurement is path integrated in nature. Therefore, if nonuniformity (in terms of temperature and/or concentration) exists along the measurement path, the TDLAS technique yields line-of-sight-integrated results, which represent complicated averages of the temperature and concentration along the path. In our test facility, the nonuniformity exists mostly along the height (i.e., the $y$ direction as shown in Fig. 1) because of the quasi-two-dimensional cavity and multihole injectors used. Along the measurement path (i.e., the $z$ direction shown in Fig. 1), the temperature and concentration of water vapor were assumed to be uniform in this work. For the wavelengths chosen here, this assumption results in a path-integrated temperature that is slightly lower than the spatial average of the temperature along the path, because of the increased sensitivity at the cooler regions of the flow [15]. The undergoing research is to quantify the uniformity along the $z$ direction by using an absorptionbased tomography technique as described in [25-27].

On the other hand, the flow is highly nonuniform along the $y$ direction, and such nonuniformity needs to be resolved to calculate the enthalpy flux and combustion efficiency. In this work, the sensor is scanned along the $y$ direction under each measurement condition to resolve the nonuniformity. Obviously, such scanning requires the flow to be steady, which was an acceptable assumption because the temperature fluctuated within $\sim 6 \%$ in these tests and, furthermore, the averaged measurements were of interest in this work.

Finally, note that, in this work, we preferred to use the direct absorption strategy among the variations of the TDLAS technique. For instance, a wellestablished variation (WMS-2 $f$ ) involves imposing a high-frequency modulation on top of the wavelength tuning, measuring the second harmonic detection of the absorption signal, and using the change in the absorption signal (instead of the absorption signal itself, as in the direct absorption strategy) to infer temperature and concentration [14,28]. This WMS$2 f$ variation of TDLAS has been demonstrated to enhance the SNR of the measurements under weak absorbance [29]. However, in this work, we preferred the direct absorption strategy for several reasons. First, the absorption signal is already sufficiently strong in this work due to the relatively strong absorption transitions shown in Table 1 and the relatively long path length in our scramjet facility $(8.5 \mathrm{~cm})$. Therefore, satisfactory SNR can be obtained with the direct absorption strategy (see Subsection 5.B for more details). Second, the direct absorption strategy enjoys simplicity of implementation and signal interpretation [30]. The direct absorption technique does not need calibration, while the WMS- $2 f$ technique usually needs to be calibrated against several parameters, including the modulation depth, detector gain, and average incident laser intensity $[14,31]$. Finally, the direct absorption technique requires a lower sampling rate than the WMS$2 f$ variation; it facilitates the analysis and storage of the data. This work involves a considerable amount of data analysis and storage, because measurements need to be simultaneously digitized at six channels, with a bandwidth of $5 \mathrm{MHz}$ at each channel, and scanned along the heights. Therefore, the lower sampling rate required by the direct absorption technique is not trivial.

\section{Sensor Implementation and Scramjet Facility}

\section{A. Sensor Implementation}

Figure $\underline{2}$ illustrates the implementation of the TDLAS system discussed in Section 2 . The system consisted of two distributed feedback diode lasers (NLK1B5EAAA, NEL), each controlled (both the injection current and temperature) independently by a diode-laser controller (ITC502, Thorlabs). The linewidth of these lasers is $10 \mathrm{MHz}$ as provided by the vendor. The injection current of both controllers was modulated by a ramp signal from a signal generator (AFG3022, Tektronix) so that the lasing wavelength scanned a spectral range of $2 \mathrm{~cm}^{-1}$ to probe the $\mathrm{H}_{2} \mathrm{O}$ absorption transitions shown in Table 1 . The wavelength of each laser was modulated at a repetition rate of $8 \mathrm{kHz}$.

The laser beams from the two lasers were combined by a $2 \times 8$ single-mode fiber coupler. The separation of the absorption signals was then achieved via a time division multiplexing (TDM) scheme, and a typical measurement of the absorption signals is shown in Fig. 3. In the TDM scheme, each laser was scanned at a rate of $8 \mathrm{kHz}$ (i.e., $0.125 \mathrm{~ms}$ per scan). During each scan, the laser current was set below the threshold for $0.025 \mathrm{~ms}$, yielding a background signal level to correct for combustion emission. Each determination of temperature and water partial pressure required scanning both transitions, as shown in Fig. 3 , resulting in a temporal response of $4 \mathrm{kHz}$ (i.e., $0.25 \mathrm{~ms}$ per measurement) if no averaging was performed.

The $2 \times 8$ fiber coupler then split the combined beam into eight channels. The first channel was delivered into a Fabry-Perot interferometer to monitor the frequency during the wavelength scanning. The second channel was registered by a detector without

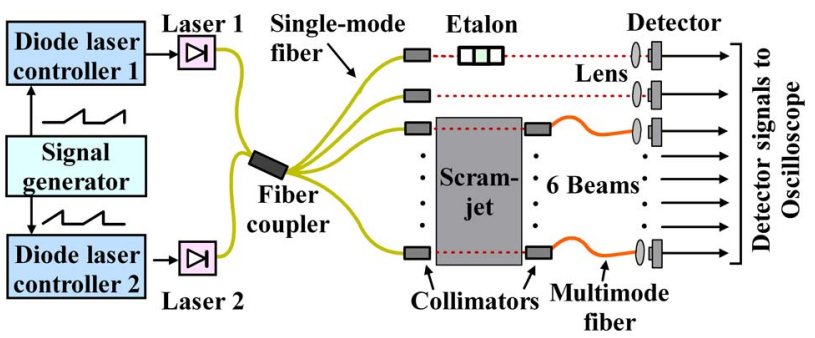

Fig. 2. (Color online) Optical layout of the TDLAS system. 


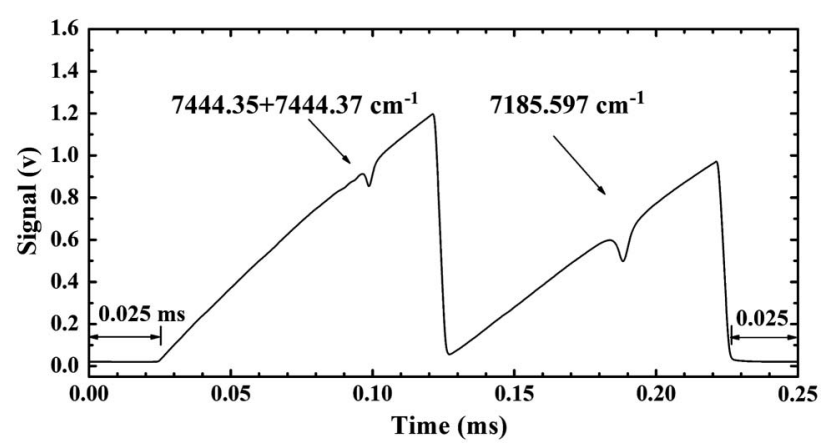

Fig. 3. Transmission signals obtained by the TDM scheme.

going through the scramjet, providing a reference of the laser intensity during scanning. Nitrogen purging was used (on all beams) to eliminate interference absorption from the water vapor in ambient air. The other six beams were the probing beams, and they were separated into three pairs and delivered to the measurement locations, as shown in Fig. 1(b). The first pair (beams 1 and 2) crossed at an angle of $\alpha_{1}=60^{\circ}$ to measure the target parameters at the inlet of the combustor; the second pair (beams 3 and beam 4) crossed at an angle of $\alpha_{2}=30^{\circ}$ for measurement at cavity 2 within the combustor; and the third pair (beams 5 and 6) crossed at an angle of $\alpha_{3}=60^{\circ}$ for measurements at the exit of the combustor. These angles were designed based on two considerations. First, they were designed to accommodate the size and geometry of the windows. Second, a larger angle was preferred at locations (windows 1 and 4) where the pressure distribution is uniform and hence presents no interference to the Doppler shift, while a smaller angle was preferred at the location (window 3 ) where significant nonuniformity in pressure was expected to minimize the interference caused by pressure shift. To prevent the etalon effects, quartz window 3 was wedged with $3^{\circ}$ on the outer surface.

On the catch side, six collimators with relatively large numerical aperture $(\mathrm{NA}=0.49)$ captured the transmitted beams into multimode fibers. Six lenses then focused the beams out of the fibers onto six InGaAs detectors with $2 \mathrm{~mm}$ diameter sensitive areas. The parameters of these collection optics were designed to minimize the effects of beam steering caused by the turbulence along the measurement path and the mechanical vibration of the scramjet engine. The relatively large numerical aperture of the collimators and the large active area of the detectors minimizes the effects of beam steering. The path length of transmitted beams in free space was minimized to reduce the effects of mechanical vibration, and the relatively high scan rate also reduces the effects of mechanical vibration. Finally, two high dynamic oscillationscopes (DPO4034, Tektronix) were employed for recording the data from the detectors at a sample rate of $5 \mathrm{MHz}$.

For overall combustion efficiency diagnostic, distributions of flow parameters along the height need to be obtained. A motorized precision translation stage was used to translate beam pair 5 and 6 across the entire height at the exit of the combustor (i.e., window 4 as shown in Fig. 1). To distinguish the combustion mode, another motorized precision translation stage translated beam pair 3 and 4 across the combusting region under cavity 2 (i.e., window 3 as shown in Fig. 1). These translation stages were moved at a speed of 80 and $40 \mathrm{~mm} / \mathrm{s}$ at windows 4 and 3 (due to the different height of these windows), respectively, so that the entire height could be scanned in a single run.

\section{B. Data Processing}

To obtain temperature and partial pressure of water vapor, the data processing involved three steps. First, for each measured absorbance signal as shown in Fig. 3 , the nonabsorbing wings were extracted and fitted into a third-order polynomial, which was used as the baseline for calculating the absorption spectra. Second, Voigt profiles were used to fit the absorption spectra obtained in the first step. The bottom panel of Fig. 4 shows an example of the Voigt fit of the absorption spectra, and the top portion shows the fitting residual, which is less than $1 \%$ of the peak absorption throughout the line profiles. Such low fitting residual illustrates the high SNR achieved in this research. Third, based on the Voigt fit obtained in the second step, the integrated absorbance was calculated, and then used to calculate the water vapor partial pressure and temperature. All these steps were automated using a computer code described in [24].

To obtain velocity measurement, the spectral position of the absorption peaks need to be accurately captured. In this work, we found that the Voigt fitting described above was not suitable for accurately determining the position of the absorption peaks. The fitting criterion of the Voigt profile was to minimize the overall residual between the fit and the measurements (thereby providing accurate determination of the integrated absorbance), and the positions of the absorption peak were not a fitting criterion. Hence, an alternative fitting algorithm was sought to determine the peak positions. In this

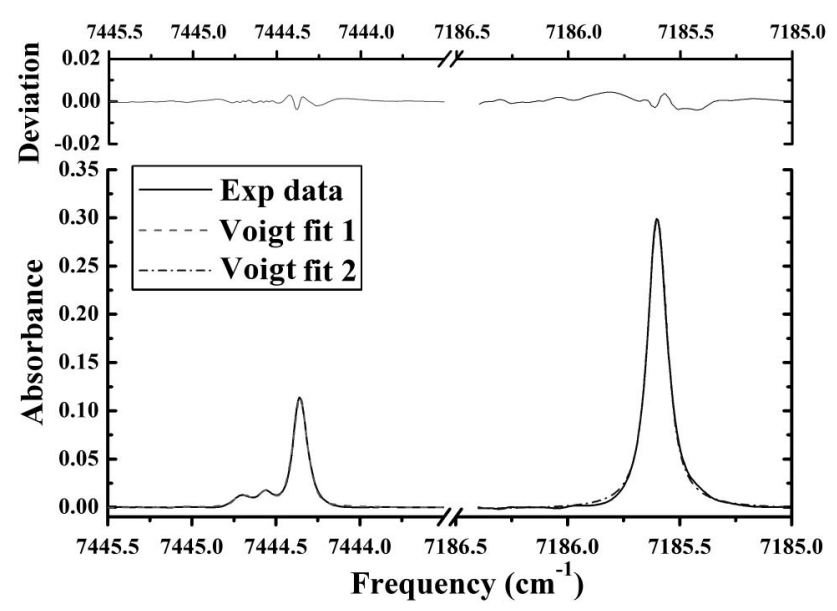

Fig. 4. Illustration of fitting of the absorption features. 
work, we found that a further fitting of the region near the peak using fourth-order polynomials resulted in accurate positions of absorption peaks. Therefore, for the velocity measurement, an additional fitting process was added into the computer code to perform a further fit of the peak region using fourth-order polynomials. Once the peak positions were determined, they were combined with the measurement from the Fabry-Perot interferometer to determine the frequency shift, which was then used in Eq. (1) to calculate velocity.

Note that, in the presence of pressure nonuniformity, the pressure-induced line shift may interfere with the Doppler shift for velocity measurement. However, for beam pair 5 and 6, the two beams experienced the same pressure drop and the pressure shift was canceled during the calculation of velocity. Therefore, the interference caused by pressureinduced shift was neglected for the measurements by beam pair 5 and 6 . As to the beam pair 3 and 4 , the pressure transducers indicated a slight unequal drop of static pressure across beams 3 and 4 , and our analysis showed that such slight unequal pressure drop only led to minor effect on the velocity measurement.

\section{Scramjet Facility}

The TDLAS system described above was applied in a direct-connected scramjet test facility operated on ethylene $\left(\mathrm{C}_{2} \mathrm{H}_{4}\right)$, as shown in Fig. 1. The operation parameters of the flow entering the combustor are summarized in Table 2.

Compressed dry air was heated in the heater, as shown in Fig. 1(a), by hydrogen combustion to obtain the desired temperature and pressure. The burned gas was then replenished with oxygen also in the heater [32]. The resulting high-enthalpy flow was accelerated to supersonic speed through a Mach 2.5 nozzle. The flow then passed though a $200 \mathrm{~mm}$ long isolator, entering the combustor with a cross-section area of $40 \mathrm{~mm} \times 85 \mathrm{~mm}$. The bottom wall of the combustor was horizontal, while the top wall had a $2^{\circ}$ divergence angle. The combustor housed two cavities (labeled cavity 1 and cavity 2 in Fig. 1) located in the top wall, both with igniters in the middle. The igniter at cavity 2 was used to ignite a hydrogen flame, which acted as a pilot flame to ignite and stabilize the $\mathrm{C}_{2} \mathrm{H}_{4}$ fuel, injected through seven injectors with a diameter of $0.8 \mathrm{~mm}$. The $\mathrm{C}_{2} \mathrm{H}_{4}$ fuel was injected into the flow through seven injectors with a diameter of $1.2 \mathrm{~mm}$ located $60 \mathrm{~mm}$ upstream cavity 1 .

The test facility was outfitted with four pair of quartz windows on the sidewalls for the optical mea-

Table 2. Operation Parameters of the Scramjet Facility in this Study

\begin{tabular}{llll}
\hline $\begin{array}{c}\text { Mach } \\
\text { Number [-] }\end{array}$ & \multicolumn{2}{c}{ Total } & \multicolumn{2}{c}{ Total } & Flow \\
& Temperature $[\mathrm{K}]$ & Pressure $[\mathrm{MPa}]$ & Rate $[\mathrm{kg} / \mathrm{s}]$ \\
\hline 2.5 & 1650 & 1.0 & 1.2 \\
\hline
\end{tabular}

surements, as shown in Fig. 1 . Window 1 was located at the end of the isolator for the measurement of the combustor inlet condition. Windows 2 and 3 were located at cavities 1 and 2 , respectively. These first three windows had the same size $(46 \mathrm{~mm}$ height by $120 \mathrm{~mm}$ width). Window 2 was intended for schlieren photography and $\mathrm{CH}^{*}$ chemiluminescence imaging and was not used in this work. Window 3 was used for the TDLAS measurement near cavity 2 in the combustor. Window 4 had a different size $(100 \mathrm{~mm}$ height by $50 \mathrm{~mm}$ width), and was located $600 \mathrm{~mm}$ downstream the $\mathrm{C}_{2} \mathrm{H}_{4}$ injectors at the exit of the combustor. Window 4 provided optical access to the entire height of the exit of the combustor. Besides the optical sensors, the isolator and combustor were also equipped with 30 static pressure transducers along the flow direction. These pressure transducers provided the static pressure for processing the TDLAS measurements.

\section{Results and Data Analysis}

This section reports the data obtained using the TDLAS system in our scramjet facility. Measurements were conducted under different operation conditions, and here we report the example results obtained under a stable operation Mach number of 2.5. Results obtained under other operation conditions were reported elsewhere [24,33]. Here, we specifically report the results obtaine at three locations: the inlet of the combustor (Subsection 4.A), near cavity 2 in the combustor (Subsection $\overline{4 . B}$ ), and at the exit of the combustor (Subsection 4.C). These results are of special importance to the development of scramjet engines for several reasons. First, the measurements at the inlet and exit can be used to infer combustion efficiency, a critical parameter. Second, the measurements in the combustor (near cavity 2) can be used to resolve the combustion mode. And last, in this work, the measurements at the combustor inlet can be compared to the design parameters of the hydrogen heater, thusly providing a verification of the TDLAS results.

\section{A. Measurements at Combustor Inlet}

Figure 5 shows a set of the static temperature, water vapor partial pressure, and velocity measured at combustor inlet. The data shown here recorded $2 \mathrm{~s}$ of measurements. The results show an average temperature of $798 \mathrm{~K}$, an average partial pressure of water vapor of $0.139 \mathrm{~atm}$, and an average velocity of $1335 \mathrm{~m} / \mathrm{s}$. The pressure transducers fixed on the bottom wall measured a static pressure of $0.61 \mathrm{~atm}$. The mole fraction of water vapor in the flow can be inferred to be $23 \%$ by combining the partial pressure and the static pressure measurement. As mentioned above, these measurements performed at the combustor inlet can be compared with the designed operating parameters of the heater. For the case shown in Fig. 5 , the difference between the designed and measure $\bar{d}$ values was $21 \mathrm{~K}$ for temperature (2.7\%), $2.9 \times 10^{-3} \mathrm{~atm}$ for $\mathrm{H}_{2} \mathrm{O}$ partial pressure 


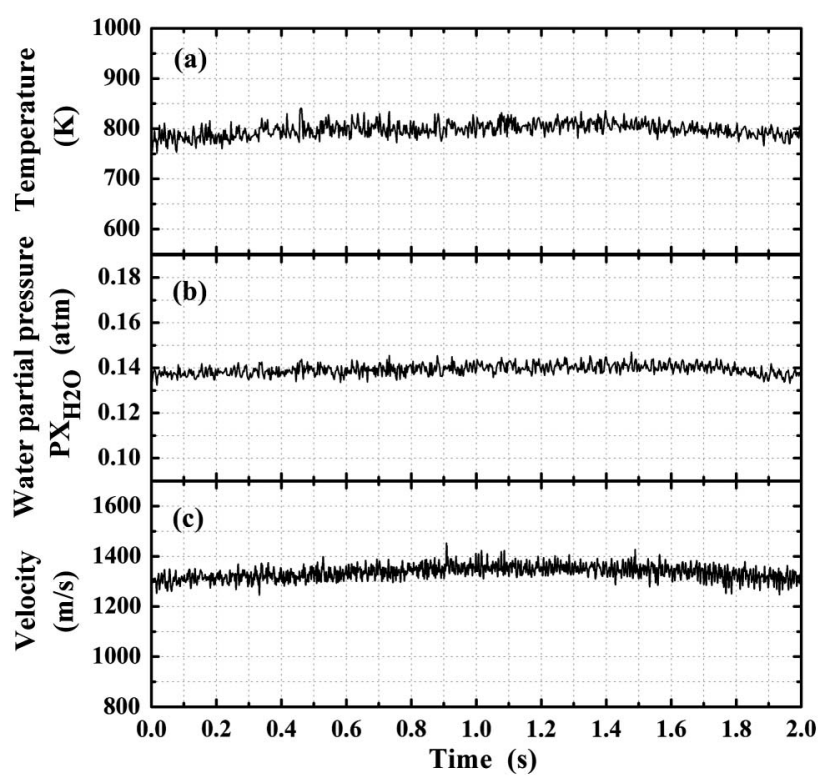

Fig. 5. Results of multiple parameters at the inlet, measured at $4 \mathrm{kHz}$ measurement and filtered to $1 \mathrm{kHz}$ in this plot. (a) Temperature, (b) water partial pressure, and (c) velocity versus time at the inlet of the combustor.

(2.2\%), and $32 \mathrm{~m} / \mathrm{s}$ for velocity (2.4\%). Such good agreement provides a validation of the application of the TDLAS system in scramjet facilities.
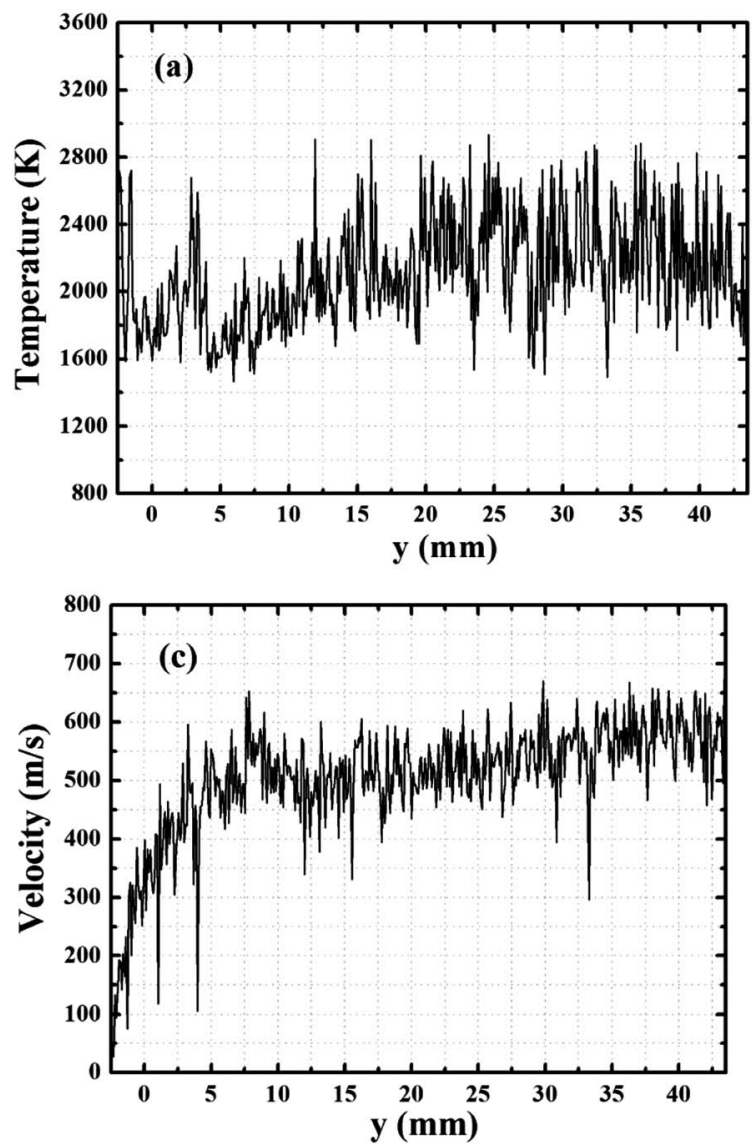

As mentioned at the beginning of section, these results were to be combined with the measurements at the combustor exit to infer the overall combustion efficiency of the scramjet engine.

\section{B. Measurements Near Cavity 2}

Figures 6(a)-6(d), respectively, show a set of example measurements of temperature, partial pressure of water vapor, velocity, and Mach number near cavity 2 in the combustor. The temperature and water vapor partial pressure were measured using beam 4 , and the velocity was measured using beams 3 and 4 . Then the Mach number was inferred from the temperature and velocity. All these measurements were scanned along the height direction so that represented spatially resolved measurements. The $y=0$ position corresponds to the position of the top wall. These measurements are important for determining the combustion mode in a scramjet engine. Note that, to our knowledge, it is the first time that the distribution of velocity measurement by the TDLAS technique in a scramjet combustor has been reported, although [34] has reported similar distribution measurements of velocity using hydroxyltagging velocimetry.

As shown in Fig. 6, the measurement results have shown spatial variation (i.e., variation in the $y$ direction). Such variation was caused partially
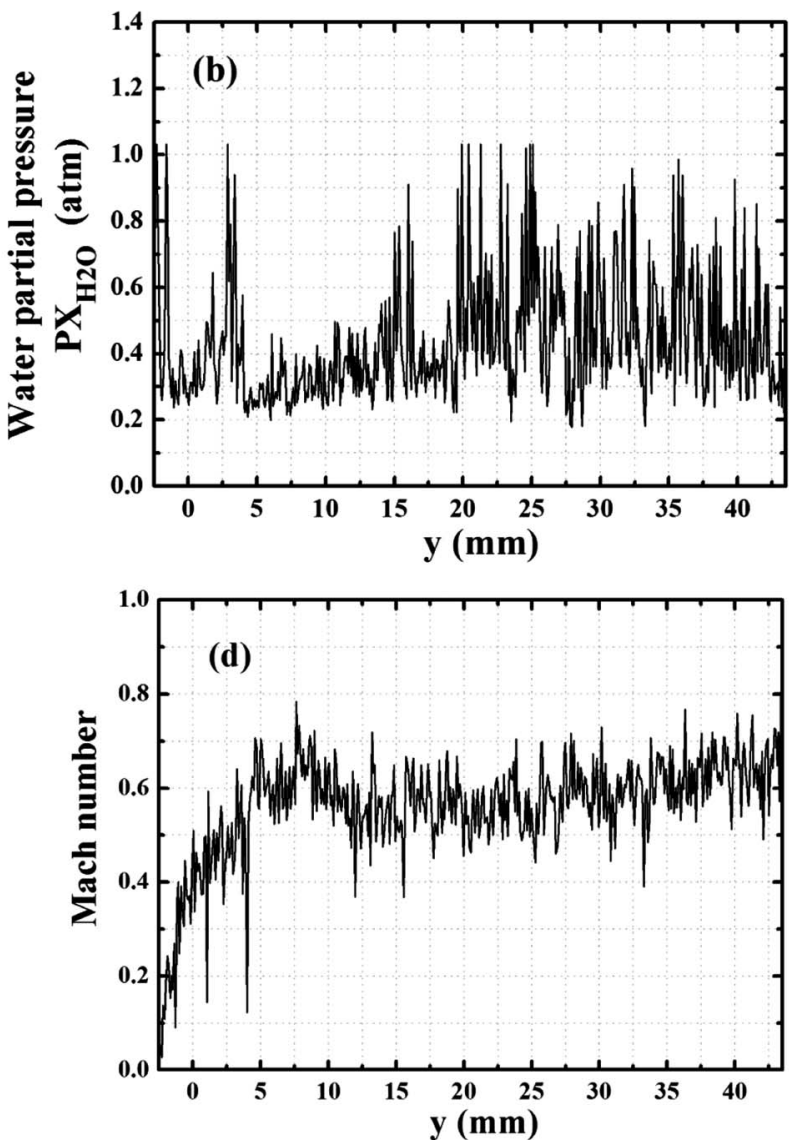

Fig. 6. Distribution of (a) static temperature, (b) partial pressure of water vapor, (c) velocity, and (d) Mach number along the vertical location near the cavity. Measured at $4 \mathrm{kHz}$ bandwidth and filtered to $1 \mathrm{kHz}$ in this plot. 
by temporal fluctuation (due to the limited temporal resolution of the sensor) and partially by spatial fluctuation (due to the actual variation of the measured quantities). Take temperature, the key parameter for the calculation of efficiency, as an example. The contribution from temporal variation was estimated to be $150 \mathrm{~K}$ at the scan rate used here, based on our previous experiments [33] taken by the TDLAS sensor at a fixed location. Each data point shown in Fig. 6 was taken in a duration of $1 \mathrm{~ms}$ (the sensor was scanned at $4000 \mathrm{~Hz}$ and each point in Fig. 6 is the average of four scans). Therefore, the probing beam translated for $0.04 \mathrm{~mm}$ during the measurement of each data point, which was much smaller than the $1 \mathrm{~mm}$ diameter of the probing beam. Furthermore, in this work, the calculation of the overall efficiency requires integrating the measurements (detailed in Section 5), so the effects of both the spatial and temporal fluctuation were expected to be averaged out.

The results in Figs. 6(a) and (b) provided a wealth of information for understanding the combustion characteristic in the combustor. From the results shown in Fig. 6(a), the average temperature across the height can be calculated to be about $2200 \mathrm{~K}$. Figure 6(a) shows that the temperature was consistently higher than this average in the region from 15 to $40 \mathrm{~mm}$, which confirmed the intensive combustion in this region caused by the high penetration depth of the fuel injection. The measurements also showed another region with temperature higher than the average: the region from the $0-4 \mathrm{~mm}$, i.e., in the shear layer of cavity 2 . The higher temperature in this region was caused by the hydrogen pilot flame. These results also illustrated that the temperature inside the cavity (i.e., the region below $0 \mathrm{~mm}$ ) was not substantially higher than the region from 15 to $40 \mathrm{~mm}$, indication that intensive combustion occurred in the core/middle region of the combustor chamber and only a small of fraction of the fuel entered and burned inside cavity 2 . The temperature measured across the entire height was significantly higher than the stagnation temperature of flow at the combustor inlet, which was designed at $1650 \mathrm{~K}$, as shown in Table 2 . Such a temperature rise across the entire height suggested that the combustion occurred across the whole engine cross section. The results of temperature combined with the partial pressure of water vapor can also provide insights into the combustion zone. For example, in the results shown in Figs. 6(a) and (b), there was increase in the partial pressure of water vapor accompanying the increase of temperature, suggesting that combustion occurred upstream of the measurement location [24].

Figure 6(c) shows the velocity distribution along the height of the section. From this figure, it can be seen that velocity decreased slightly from the bottom wall toward the upper wall, i.e., from 43.5 to $7 \mathrm{~mm}$ (note that $y=0 \mathrm{~mm}$ corresponds to the position of the upper wall). Then velocity decreased rapidly in the shear layer and in the cavity, i.e., from 7 to $-2.5 \mathrm{~mm}$. This variation of the velocity profile indicated that the thickness of the shear layer was $\sim 7 \mathrm{~mm}$ at this location. Note that a boundary layer also exists near the bottom wall, where a similar sharp velocity decrease is expected. However, this set of measurements was not able to resolve the bottom boundary layer, because the height of window 3 was smaller than height of the engine cross section, resulting in a limited field of view at this location. By combining the results shown in Figs. 6(a) and (c), the distribution of the Mach number can be calculated and is shown in Fig. 6(d). The results shown in Fig. 6(d) indicate a subsonic combustion with a peak Mach number of 0.7 .

\section{Measurements at Combustor Exit}

Figures 7(a)-7(d), respectively, show a set of example measurements of temperature, partial pressure of water vapor, velocity, and Mach number at the combustor exit. Similar to the results shown in Fig. $\underline{6}$, these measurements were obtained by scanning beams 5 and 6 in the height direction to resolve the distribution of these parameters along the height direction. The $y=0$ position again corresponds to the position of the top wall.

Compared to the results shown in Fig. 6, the overall distribution of temperature and water vapor at the combustor exit was noticeably more uniform due to convective heat and mass transfer, although the results shown in Figs. 7(a) and (b) exhibited higherthan-average temperature and water vapor partial pressure in the same regions as those observed in Figs. 6(a) and (b).

Figures $7(\mathrm{c})$ and (d) show the velocity and Mach number distribution at the combustor exit. In contrast to the results shown in Fig. 6 , the results shown here provide complete information across the entire height of the measurement cross section $(88 \mathrm{~mm})$, because the height of window 4 matched the height of the cross section. As expected, the velocity varied slightly in the main flow, and decreased sharply in the boundary layer near the top and bottom walls. Figure 7(d) shows the distribution of the Mach number, again calculated by combining the temperature measurements shown in Fig. 7(a) and the velocity measurement shown in Fig. 7(c). Figure 7(d) shows a relatively uniform Mach number of 2 in the core flow. Because the flow near cavity 2 was subsonic, as shown in Fig. 6(d), the supersonic flow at the combustor exit indicated that there was a thermal nozzle between cavity 2 and the combustor exit as expected.

Finally, as mentioned at the beginning of this section, the measurements obtained at the combustor inlet and exit can be combined to calculate the overall combustion efficiency, which is discussed immediately below in Subsection 5.A.

\section{Discussions}

\section{A. Calculation of Combustion Efficiency}

The overall combustion efficiency $\left(\eta_{C}\right)$ is a key parameter for the development and design of scramjet 

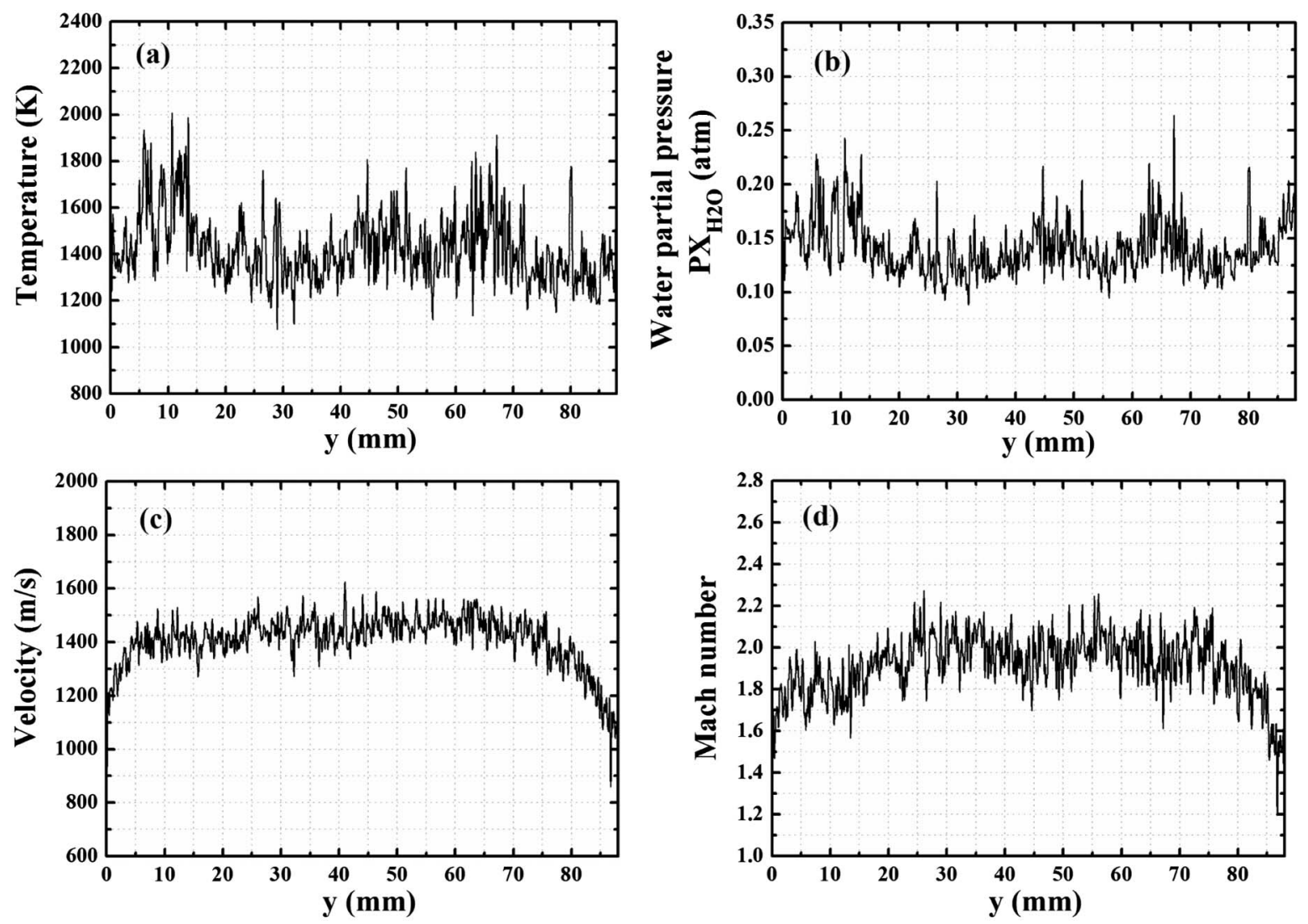

Fig. 7. Distribution of (a) static temperature, (b) partial pressure of water vapor, (c) velocity, and (d) Mach number along the vertical location at the combustor exit. Measured at $4 \mathrm{kHz}$ bandwidth and filtered to $1 \mathrm{kHz}$ in this plot.

engines. Two common methods exist for determining $\eta_{C}$ experimentally [5, 35$]$, and the TDLAS measurements described above are valuable for both methods. Thus, this section discusses the application of our TDLAS measurements for calculating $\eta_{C}$. Applications in both methods were demonstrated and the results shown to agree reasonably.

In this work, $\eta_{C}$ is defined as the fraction of fuel that is completed burnt and converted into $\mathrm{CO}_{2}$. Following this definition, the following global reaction describes the fuel consumption:

$$
\begin{aligned}
& \phi_{i} \cdot \alpha \mathrm{C}_{2} \mathrm{H}_{4}+3\left(\alpha \mathrm{O}_{2}+\beta \mathrm{H}_{2} \mathrm{O}+\chi \mathrm{N}_{2}\right) \\
& \rightarrow \eta_{c} \cdot 2 \phi_{i} \alpha \mathrm{CO}_{2}+\left(\eta_{c} \cdot 2 \phi_{i} \alpha+3 \beta\right) \mathrm{H}_{2} \mathrm{O} \\
& \quad+\phi_{i} \alpha\left(1-\eta_{c}\right) \mathrm{C}_{2} \mathrm{H}_{4}+3\left(\alpha-\eta_{c} \phi_{i} \alpha\right) \mathrm{O}_{2}+3 \chi \mathrm{N}_{2}
\end{aligned}
$$

where $\phi_{i}$ represents the equivalence ratio of $\mathrm{C}_{2} \mathrm{H}_{4}$, and $\alpha, \beta$, and $\chi$ refer to the mole fractions of $\mathrm{O}_{2}$, $\mathrm{H}_{2} \mathrm{O}$, and $\mathrm{N}_{2}$ entering the combustor, respectively.

Note that Eq. (2) assumes that (1) the hydrogen from the heater and pilot flame is completely burnt into $\mathrm{H}_{2} \mathrm{O}$, and (2) $\mathrm{CO}_{2}$ and $\mathrm{H}_{2} \mathrm{O}$ are the only products produced from the combustion of the fuel.

The first method for calculating $\eta_{C}$ relies on the amount of water vapor present at the combustor inlet and exit. Based on the assumptions made in Eq. (2), the increase in the amount of water from the inlet to the exit is solely due to the combustion of the fuel, resulting in the following equation:

$$
\begin{aligned}
\frac{1}{H} & \int_{0}^{H} \frac{P_{\mathrm{H}_{2} \mathrm{O}}(y)}{P} \mathrm{~d} y-\frac{\bar{P}_{\mathrm{H}_{2} \mathrm{O}, \text { inlet }}}{P_{\text {inlet }}} \\
& =\frac{2 \eta_{c} \phi_{i} \alpha+3 \beta}{\phi_{i} \alpha+3(\alpha+\beta+\chi)}+C_{\mathrm{H}_{2}},
\end{aligned}
$$

where $H$ is the height of the cross section, $P_{\mathrm{H}_{2} \mathrm{O}}(y)$ is the local water partial pressure at the exit, $\bar{P}_{\mathrm{H}_{2} \mathrm{O} \text {,inlet }}$ is the average water partial pressure at the inlet, $P$ and $P_{\text {inlet }}$, respectively, are the wall static pressure at the exit and the inlet, and $C_{\mathrm{H}_{2}}$ is the correction coefficient of water concentration caused by the combustion of the hydrogen pilot flame.

On the left-hand side of Eq. (3), the first term represents the amount of water vapor present at the combustor exit, and the second term presents the amount at the combustor inlet. The difference between the inlet and exit depends on the completeness of the fuel [i.e., the first term on the right-hand side of Eq. (3)] and the water vapor produced due to the hydrogen pilot flame (i.e., $C_{\mathrm{H}_{2}}$ ). In Eq. (3), the distribution of water vapor at the inlet is considered to be uniform and, therefore, the calculation of the amount of water present at the inlet is simplified. However, the calculation of the amount of water vapor at the exit involves an integration (first term on the lefthand side) due to the significant nonuniformity caused by the combustion.

In the second method, $\eta_{C}$ is calculated by the following equation: 


$$
\eta_{c}=\Delta Q_{C} / \Delta Q\left(\eta_{c}=1\right),
$$

where $\Delta Q_{c}$ and $\Delta Q\left(\eta_{C}=1\right)$ are, respectively, the actual heat release and the heat release with complete combustion (i.e., with $\eta_{C}=1$ ). It is straightforward to calculate the latter term because the amount of $\mathrm{C}_{2} \mathrm{H}_{4}$ fuel injected into the combustor was metered. The former term can be determined from the following equations:

$$
\begin{gathered}
\Delta Q_{C}=\left[\Delta h+\frac{w}{H} \int_{0}^{H} \frac{V_{e}(y)^{2}-V_{\infty}^{2}}{2} \mathrm{~d} y\right] \\
+\left[h_{f, T_{\infty}}-h_{f, i}+w_{f} \cdot \frac{V_{\infty}^{2}-V_{i}^{2}}{2}\right]+\dot{q}_{w} \\
\Delta h=\frac{w}{H} \int_{0}^{H} \int_{T_{\infty}}^{T(y)} C_{p}(T) \mathrm{d} T \mathrm{~d} y \\
\dot{q}_{w}=\frac{0.0287 p_{e} V_{\infty}\left(h_{a w}-h_{w}\right)}{R_{m} T^{*} \operatorname{Pr}^{* \frac{2}{5}} \operatorname{Re}_{x}^{* \frac{1}{5}}}
\end{gathered}
$$

where $\Delta Q_{c}$ is the heat release of combustion (i.e., the heating value of the fuel at fixed temperature $T_{\infty}$ ), $\Delta h$ is the static-enthalpy variation of the burnt gas, $w$ is the mass flow rate of the burnt gas, $H$ is the height of the cross section, $V_{e}$ is the local velocity of the flow at the exit, $V_{\infty}$ is the flow velocity at the inlet, $h_{f, T_{\infty}}$ and $h_{f, i}$ are, respectively, the static enthalpy of fuel at temperature $T_{\infty}$ and at the fuel injectors, $w_{f}$ is the mass flow rate of the injected fuel, $V_{i}$ is the velocity of the fuel at the fuel injectors, $C_{p}(T)$ is the specific heat capacity, and $\dot{q}_{w}$ is the heat loss through the wall surface.

Equation (7) represents an estimation of the heat loss through the wall surface following the Eckert reference enthalpy method [36], where $p_{e}$ refers to the static pressure at the inlet of the combustor, $h_{a w}$ refers to the stagnation enthalpy of the flow, $h_{w}$ is the static enthalpy of the flow at the wall temperature, $R_{m}$ is the universal gas constant, $T^{*}$ is the reference temperature, $\mathrm{Pr}^{*}$ is the Prandtl number at the reference temperature, and $\mathrm{Re}_{x}^{*}$ is the Reynolds number at the reference temperature.

Equation (5) essentially represents the conversation of energy of the flow through the combustor: the heat release of combustion (at the fixed temperature $T_{\infty}$ ) is equal to the summation of (1) the stagnation-enthalpy increase of the burnt gas due to the variation of temperature and velocity, (2) the stagnation-enthalpy increase of the fuel during the residence time (from injection to combustion), and (3) the heat loss at wall surface.

The first method relies on the amount of water vapor present at the combustor inlet and exit to calculate $\eta_{C}$. The amount of water vapor at both the inlet and exit was calculated using the static pressure (measured by pressure transducer) and the partial pressure of water vapor (measured by the TDLAS system). Under the operation conditions listed in Table 2 , at the inlet, the static pressure was measured to be $0.61 \mathrm{~atm}$ and the partial pressure of water vapor $0.139 \mathrm{~atm}$; at the exit, the static pressure was measured to be $0.58 \mathrm{~atm}$ and the average partial pressure of water vapor [i.e., the first term on the left-hand-side of Eq. (3)] was determined to be $0.141 \mathrm{~atm}$ using the results shown in Fig. 7(b). Based on these, $\eta_{C}$ was calculated to be $76.2 \%$.

In the second method, the required inputs included the equivalence ratio $\left(\phi_{i}=0.54\right)$, the temperature and velocity distribution along the vertical location at the combustor exit [as shown in Figs. 7(a) and (c)], and the wall temperature $(800 \mathrm{~K}$, for evaluation of static enthalpy $h_{w}$ ). Based on these inputs, $\eta_{c}$ was determined as $81.5 \%$, which was in reasonable agreement with the results obtained from the first method. Also, these calculations also indicated that the heat loss through the wall counted for $15 \%$ of the total combustion heat release, justifying the consideration of the heat release in the calculation. Further discussion on the calculation of the combustion efficiency and the heat loss through wall surface based on the TDLAS measurements will be reported elsewhere.

\section{B. Measurement Uncertainty}

The high signal strength obtained in this study yielded measurements with relatively high SNR, as indicated by the small fitting residual shown in Fig. 4. If the SNR is defined as the ratio between the maximum absorbance at the line center and the maximum fluctuation at the nonabsorption wings, then the SNR for our measurements ranged from 20 inside the combustor (beam pair 3 and 4 ) to $\sim 30$ at the combustor inlet (beam pair 1 and 2). Under such conditions, the error in the integrated absorbance was dominated by the fitting error of the base line [37]. The standard error, defined as the standard deviation divided by the square root of the sampling number, was found to be about $1.2 \times$ $10^{-4}$ in the nonabsorbing wings when the baseline was fitted to a third-order polynomial. Such a standard error translated into an error of $\sim 1 \%$ in the integrated absorbance and an error of $\sim 2 \%$ in the ratio of the integrated absorbance between two transitions. The $2 \%$ error in the ratio can be further translated into the error in temperature and partial pressure of water vapor. At the inlet, where the average temperature was about $800 \mathrm{~K}$, the measurement error was determined to be $12 \mathrm{~K}$ (i.e., 1.5\%) for temperature and about $0.3 \%$ for water vapor partial pressure. At the exit section, where the average temperature was about $1500 \mathrm{~K}$, the measurement error was about $40 \mathrm{~K}(2.7 \%)$ for temperature and $4.7 \%$ for water vapor partial pressure. In the combustor, where the average temperature was about $2200 \mathrm{~K}$, the measurement error was about $84 \mathrm{~K}(3.8 \%)$ for temperature and $9.8 \%$ for water vapor partial pressure. 
Using the particular absorption line pair chosen for this work, the measurement of water partial pressure has a higher error than that of temperature at temperatures higher than $\sim 1300 \mathrm{~K}$. Another line pair with different lower state energy can be used to increase the accuracy of water partial pressure in the combustion zone if desired.

The error in velocity measurement depends both on the accuracy of locating the absorption peaks and the angle at which the probe beams are crossed. The uncertainty in locating the absorption peaks was found to be $\sim 3 \times 10^{-4} \mathrm{~cm}^{-1}$. Considering the different crossing angles of the beam pairs at different measurement locations, the error in velocity measurement were calculated to be $\sim 28 \mathrm{~m} / \mathrm{s}(2.1 \%)$ at the inlet, $\sim 34 \mathrm{~m} / \mathrm{s}(2.4 \%)$ at the exit, and $\sim 48 \mathrm{~m} / \mathrm{s}$ $(8.7 \%)$ in the combustor.

\section{Summary and Outlook}

A TDLAS system was constructed and demonstrated for scramjet combustor diagnostics. This system utilized multiple measurement channels to simultaneously measure several important flow parameters (including temperature, water vapor partial pressure, and velocity) at three different locations (at the inlet and exit of the combustor, and also inside the combustor). The TDLAS system was also scanned along the height direction to resolve the nonuniformity of the flow and to obtain the distribution of the flow parameters at the combustor exit and near the combustion cavity.

These measurements are of special importance for the development and optimization of scramjet engines. First, the simultaneous measurements of multiple flow parameters near the combustor cavity provide a wealth of information to resolve the combustion mode and flame zone characteristics, a critical aspect of the development of scramjet engines. Second, the distribution measurements at the combustor inlet and exit provide valuable information for characterizing the overall combustion efficiency. Last, small discrepancy between the measurements at the combustor inlet and the design parameters of the hydrogen heater provides a verification of the TDLAS results presented in this paper.

Finally, as demonstrated in this work, the relatively high SNR of the raw signals and the high scan rate of the TDLAS system show promise to overcome the measurement challenges encountered in scramjet engines. Because of these unique virtues, we expect the TDLAS system to play an important role in the development of scramjet engines. Our ongoing work includes the expansion into multiple spectral features and a multibeam system to obtain tomographic imaging to resolve nonuniformity along the line of sight.

The work is partially supported by National Natural Science Foundation of China (Grant Nos. 90816015 and 11002148). The authors acknowledge the technical assistance provided by the techni- cians D. H. Song and Z. M. Luo at the Institute of Mechanics, Chinese Academy of Sciences.

\section{References}

1. E. T. Curran, "Scramjet engines: the first forty years," J. Propul. Power 17, 1138-1148 (2001).

2. W. H. Heiser and D. T. Pratt, Hypersonic Airbreathing Propulsion, AIAA Education Series (AIAA, 1994).

3. R. A. Baurle and D. R. Eklund, "Analysis of dual-mode hydrocarbon scramjet operation at Mach 4-6.5," J. Propul. Power 18, 990-1002 (2002).

4. T. Kanda, N. Chinzei, K. Kudo, and A. Murakami, "Dualmode operations in a scramjet combustor," J. Propul. Power 20, 760-763 (2004).

5. J. C. McDaniel, C. P. Goyne, E. B. Bryner, D. B. Le, C. T. Smith, and R. H. Krauss, "Dual-mode scramjet operation at a Mach 5 flight enthalpy in a clean air test facility," AIP Conf. Proc. 762, 1277-1282 (2005).

6. D. J. Micka and J. F. Driscoll, "Combustion characteristics of a dual-mode scramjet combustor with cavity flameholder," Proc. Combust. Inst. 32, 2397-2404 (2009).

7. T. Mitani and T. Kouchi, "Flame structures and combustion efficiency computed for a Mach 6 scramjet engine," Combust. Flame 142, 187-196 (2005).

8. M. P. Arroyo, S. Langlois, and R. K. Hanson, "Diode laser absorption technique for simultaneous measurements of multiple gasdynamic parameters in high-speed flows containing water-vapor," Appl. Opt. 33, 3296-3307 (1994).

9. A. Mohamed, B. Rosier, D. Henry, Y. Louvet, and P. L. Varghese, "Tunable diode laser measurements on nitric oxide in a hypersonic wind tunnel," AIAA J. 34, 494-499 (1996).

10. M. G. Allen, "Diode laser absorption sensors for gas-dynamic and combustion flows," Meas. Sci. Technol. 9, 545-562 (1998).

11. B. L. Upschulte, M. F. Miller, and M. G. Allen, "Diode laser sensor for gasdynamic measurements in a model scramjet combustor," AIAA J. 38, 1246-1252 (2000).

12. E. B. Bryner, G. S. Diskin, C. P. Goyne, J. C. McDaniel, R. H. Krauss, and T. A. Slate, "Water vapor concentration measurement in high enthalpy flows using infrared absorption," in Proceedings of Thirty-Ninth American Institute of Aeronautics and Astronautics / American Society of Mechanical Engineers / Society of Automotive Engineers /American Society for Engineering Education Joint Propulsion Conference and Exhibit (American Institute of Aeronautics and Astronautics, 2003), AIAA 2003-4580.

13. A. D. Griffiths and A. F. P. Houwing, "Diode laser absorption spectroscopy of water vapor in a scramjet combustor," Appl. Opt. 44, 6653-6659 (2005).

14. J. T. C. Liu, G. B. Rieker, J. B. Jeffries, M. R. Gruber, C. D. Carter, T. Mathur, and R. K. Hanson, "Near-infrared diode laser absorption diagnostic for temperature and water vapor in a scramjet combustor," Appl. Opt. 44, 6701-6711 (2005).

15. M. Gruber, C. Carter, and M. Ryan, "Laser-based measurements of $\mathrm{OH}$, temperature and water vapor concentration in a Hydrocarbon-fueled scramjet," in Proceedings of FortyFourth American Institute of Aeronautics and Astronautics / American Society of Mechanical Engineers / Society of Automotive Engineers/American Society for Engineering Education Joint Propulsion Conference and Exhibit (American Institute of Aeronautics and Astronautics, 2008), AIAA 2008-5070.

16. C. D. Lindstrom, K. R. Jackson, S. Williams, R. Givens, W. F. Bailey, C. J. Tam, and W. F. Terry, "Shock-train structure resolved with absorption spectroscopy Part 1: system design and validation," AIAA J. 47, 2368-2378 (2009).

17. G. B. Rieker, J. B. Jeffries, R. K. Hanson, T. Mathur, M. R. Gruber, and C. D. Carter, "Diode laser-based detection of combustor instabilities with application to a scramjet engine," Proc. Combust. Inst. 32, 831-838 (2009).

18. L. C. Philippe and R. K. Hanson, "Laser diode wavelength modulation spectroscopy for simultaneous measurement of temperature, pressure and velocity in shock-heated oxygen flows," Appl. Opt. 32, 6090-6103 (1993). 
19. J. A. Silver, D. J. Kane, and P. S. Greenberg, "Quantitative species measurements in microgravity flames with near-IR diode lasers," Appl. Opt. 34, 2787-2801 (1995).

20. M. Brown and T. Barhorst, "Post-flight analysis of the diode laser based mass capture experiment onboard HIFiRE flight1," in Proceedings of Seventeenth AIAA International Space Planes and Hypersonic Systems and Technologies (American Institute of Aeronautics and Astronautics, 2011), AIAA 2011-2359.

21. L. S. Rothman, I. E. Gordon, A. Barbe, D. C. Benner, P. F. Bernath, M. Birk, V. Boudon, L. R. Brown, A. Campargue, J. P. Champion, K. Chance, L. H. Coudert, V. Dana, V. M. Devi, S. Fally, J. M. Flaud, R. R. Gamache, A. Goldman, D. Jacquemart, I. Kleiner, N. Lacome, W. J. Lafferty, J. Y. Mandin, S. T. Massie, S. N. Mikhailenko, C. E. Miller, N. Moazzen-Ahmadi, O. V. Naumenko, A. V. Nikitin, J. Orphal, V. I. Perevalov, A. Perrin, A. Predoi-Cross, C. P. Rinsland, M. Rotger, M. Šimečková, M. A. H. Smith, K. Sung, S. A. Tashkun, J. Tennyson, R. A. Toth, A. C. Vandaele, and J. VanderAuwera, "The HITRAN 2008 molecular spectroscopic database," J. Quant. Spectrosc. Radiat. Transfer 110, 533-572 (2009).

22. S. T. Sanders, J. A. Baldwin, T. P. Jenkins, D. S. Baer, and R. K. Hanson, "Diode-laser sensor for monitoring multiple combustion parameters in pulse detonation engines," Proc. Combust. Inst. 28, 587-594 (2000).

23. X. Zhou, X. Liu, J. B. Jeffries, and R. K. Hanson, "Development of a sensor for temperature and water concentration in combustion gases using a single tunable diode laser," Meas. Sci. Technol. 14, 1459-1468 (2003).

24. F. Li, X. Yu, H. Gu, Z. Li, L. Chen, and X. Chang, "Measurement of temperature, velocity and water vapor concentration in a scramjet combustor based on near-infrared diode laser absorption," in Proceedings of Seventeenth AIAA International Space Planes and Hypersonic Systems and Technologies (American Institute of Aeronautics and Astronautics, 2011), AIAA 2011-2214.

25. W. Cai, D. J. Ewing, and L. Ma, "Application of simulated annealing for multispectral tomography," Comput. Phys. Commun. 179, 250-255 (2008).

26. S. T. Sanders, J. Wang, J. B. Jeffries, and R. K. Hanson, "Diodelaser absorption sensor for line-of-sight gas temperature distributions," Appl. Opt. 40, 4404-4415 (2001).
27. X. Liu, J. B. Jeffries, and R. K. Hanson, "Measurement of nonuniform temperature distributions using line-of-sight absorption spectroscopy," AIAA J. 45, 411-419 (2007).

28. G. B. Rieker, H. Li, X. Liu, J. B. Jeffries, R. K. Hanson, M. G. Allen, S. D. Wehe, P. A. Mulhall, and H. S. Kindle, "A diode laser sensor for rapid, sensitive measurements of gas temperature and water vapour concentration at high temperatures and pressures," Meas. Sci. Technol. 18, 1195-1204 (2007).

29. R. K. Hanson and J. B. Jeffries, "Diode laser sensor for ground testing," in Proceedings of Twenty-fifth Aerodynamic Measurement Technology and Ground Testing Conference (American Institute of Aeronautics and Astronautics, 2006), AIAA 2006-3441.

30. J. A. Silver and D. J. Kane, "Diode laser measurements of concentration and temperature in microgravity combustion," Meas. Sci. Technol. 10, 845-852 (1999).

31. X. Liu, J. B. Jeffries, R. K. Hanson, K. M. Hinckley, and M. A. Woodmansee, "Development of a tunable diode laser sensor for measurements of gas turbine exhaust temperature," Appl. Phys. B 82, 469-478 (2006).

32. H. Gu, L. Chen, and X. Chang, "Experimental investigation on the cavity-based scramjet model," Chin. Sci. Bull. 54, 2794-2799 (2009).

33. F. Li, X. Yu, H. Gu, Z. Li, L. Chen, and X. Chang, "Measurement of flow parameters in a scramjet combustor based on near-infrared absorption," Chin. J. Theor. Appl. Mech. 43, 1-7 (2011) (in Chinese).

34. M. D. Lahr, R. W. Pitz, Z. W. Douglas, and C. D. Carter, "Hydroxyl-tagging-velocimetry measurements of a supersonic flow over a cavity," J. Propul. Power 26, 790-797 (2010).

35. Y. Pan, Z. G. Wang, and W. D. Liu, "Introduction of scramjet combustion efficiencies measurement methods," J. Exp. Fluid Mech. 21, 68-73 (2007) (in Chinese).

36. E. R. G. Eckert, "Engineering relations for heat transfer and friction in high-velocity laminar and turbulent boundary layer flow over surfaces with constant pressure and temperature," Trans. ASME 78, 1273-1284 (1956).

37. J. T. C. Liu, J. B. Jeffries, and R. K. Hanson, "Wavelength modulation absorption spectroscopy with $2 f$ detection using multiplexed diode lasers for rapid temperature measurements in gaseous flows," Appl. Phys. B 78, 503-511 (2004). 